\title{
Sentinel Lymph Node Biopsy in Cervical Cancer
}

Carcinoma cervix is a common malignancy among females in developing countries., During the past decade chemoradiation has emerged as the treatment of choice for locally advanced cervical cancer: Surgery plays a major role in the management of early stage cervical cancer ( IA2 -II A ) and pelvic lymphadenectomy is a key component of radical surgery for cervical cancer.

There have been controversies regarding the prognostic and therapeutic significance of lymphadenectomy. There are two schools of thought - one believing that lymph node metastases is an indicator of survival and the other believing that lymph node metastases is a governor of survival. If lymph node metastasis is an indicator of survival, the removal of affected nodes will not improve survival where as, if lymph node metastasis is a governor of survival, complete lymphadenectomy is justified in all the patients with positive nodes. As far as routine clinical practice is concerned, together with parametrial extension and positive surgical margins, one of the most important prognostic factors in cervical cancer is lymph node metastasis. According to the published literature, the incidence of pelvic lymph node metastasis in stage Ib, stage IIa, and stage IIb cervical cancer ranges from 10 to $40 \% .2^{2+}$ The risk of lymph node metastasis is related to various histopathological features , including tumor size, stromal invasion, microscopic parametrial involvement, and lymphovascular invasion. The 5-year survival of pelvic node positive patients with stage Ib-IIb cervical cancer ranges from $64 \%$ to $68.2 \%$. 5.6 The survival of node positive patients is further affected by the site and the number of positive nodes. Inoue et al reported the effect of the number of positive nodes on stage Ib to IIb cervical cancer. The 5-year survival rate for node-negative patients, patients with one positive node, those with two to three positive nodes, and those with four or more positive nodes was $89 \%, 81 \%, 41 \%$, and $23 \%$, respectively. Sakuragi et $\mathrm{l}^{6}$ reported 5 year survival rate of $84.9 \%$ for patients with one positive node and $26.5 \%$ for patients with two or more positive nodal groups. Survival of patients with one positive node was not different from the survival of patients with no positive node. Common iliac node metastasis and paraaortic node metastasis are associated with extremely poor survival ranging from $33.3 \%$ to $35 \%, 9,10$

Ideally adjuvant treatment decisions should be tailored according to the individual patients risk factors. Patients with limited nodal involvement will benefit from adjuvant radiotherapy and patients with paraaortic lymph node involvement may require post operative chemoradiotherapy in view of dismal survival reported with surgery and radiotherapy alone. Where as patients with negative or single positive node may not require adjuvant therapy at all .

Various imaging modalities have been tried to assess lymphatic spread in cervical cancer. Magnetic resonance imaging (MRI) and computed tomography (CT) are often used in preoperative assessment. Because the size of the nodes on imaging does not correlate well histopathological findings, imaging techniques have limitations in diagnosing microscopic lymph node metastasis. In a review, Boss et al" reported a mean accuracy of $86 \%$ (range, $75 \%$ to $100 \%$ ) for lymph node metastasis detection by MRI and the mean percentage of over staging was $4 \%$ and under staging was $13 \%$. The mean accuracy of lymph node detection by CT scan was $81 \%$ (range, $75 \%$ to $86 \%$ ). In another review, Bipat et al $^{12}$ reported a higher sensitivity of MRI for lymph node involvement in comparison to CT, but the sensitivities were only $60 \%$ for MRI and $43 \%$ for CT Scan. Recently positron emission tomography (PET) scan is being investigated for staging cervical cancer. Chou et $\mathrm{al}^{13}$ investigated the clinical benefit of PET in 
sixty patients with stage Ia2 - IIa cervical cancer with MRI defined negative nodal metastasis. A preoperative PET scan was performed in all the patients. Final histopathological assessment revealed lymph node metastases in 10 patients and PET scan could detect only 1 out of 10 . suggesting that PET has limitations in primary, nonbulky, stage Ia2-IIa lymph node-negative cervical cancer:

Recently the sentinel node biopsy (SLNB) is emerging as a minimally invasive alternative to systematic lymphadenectomy for staging in various solid tumors especially in melanoma and breast cancer. In the gynecologic oncology field sentinel lymph node biopsy was first tried in vulvar ${ }^{14}$ and endometrial cancer. ${ }^{\text {t5 }}$ Reports of Studies on sentinel node mapping in cervical cancer have recently been published. ${ }^{16 \cdot 19}$ However, majority are single institutional studies with small sample size. Before we introduce sentinel node biopsy into routine clinical practice, there is a need to know the detection rate of sentinel nodes, the accuracy of procedure, false negative rates and the technique of choice for lymphatic mapping in cervical cancer, Currently, there is no clear consensus regarding the optimal technique (blue dye or radio isotope or a combination of both) or the usefulness of sentinel node procedure in cervical cancer. Published litelgture with either laparoscopic or open surgery have shown a detection rate of $80 \%$ to $100 \%$, with a false negative rate of $0 \%$ to $16 \%{ }^{18-19}$ Combinations of $99 \mathrm{~m}$ TC labeled colloid and blue dye techniques may increase the detection rate, Various methods of histopathological assessment of sentinel lymph node are described including conventional techniques, serial sectioning, RT-PCR , immunohistochemical analysis, and human papilloma virus detection. However, there is no consensus on the method of choice. One of the main aims of sentinel lymph node biopsy is to decrease the morbidity of lymphadenectomy but in general the morbidity of pelvic lymphadenectomy is less debilitating than the morbidity of axillary lymph node dissection . Hence, most probably the role of SLNB in cervical cancer will mainly be limited to improve the staging accuracy and to avoid unnecessary lymphadenectomy in node negative patients. Manoj Babu et al from outh India have reported encouraging results of a pilot study However, further studies and standardization of techniques are required in the field of cervical cancer before its routine use can be recommended. Cancer centers from developing countries with significant cervical cancer burden must take the initiative to launch a large multi center trial to resolve the above issues.

\section{REFERENCES ;}

i. A. Nandkumar, P.C. Gupta, P. Gangadharan. PN. Visweswara, ICMR-NCGP Development of an atlas of cancer in Indla. First All Indfa Report : 2001-2002

2. Tanaka Y, Sawada S, Murata T. Relationship between lymph node metastases and prognosis in patjents irradiated postoperatively for carcinoma of the uterine cervix. Acta Radiol Oncol 1984:23:455-459

3. Inoue $T$, Morita $K$. The prognostic signifl cance of number of positive nodes in cervical carcinoma stages IB. IIA, and IIB, Cancer 1990;65:1923-1927.

4. Lee KB, Lee JM, Park CY. Lymph node metastasis and lymph vascular space invasion in microftrasive squamous cell carcinoma of the uterine cervix. Int $f$ Gynecol Cancer 2006:16: 1184-1187.

5. Garipagaoglu $M$, Tulunay $G$, Kose $M F$, et al. Prognostic factors in stage IB-IIA cervical carcinomas treated with postoperative radiotherapy. Eur d Gynaecol Oncol 1999;20:131-135.

6. Lai $\mathrm{CH}$, Hong JH. Hsueh $S$, et al. Preoperative prognostic variables and the impact of postoperative adjuvant therapy on the outcomes of stage $I B$ or $I I$ cervical carcinoma patients with or without pelvic Jymph node metastasis, Cancer 1999;85:1537-1546.

7. Inoue $T$, Morita $K$. The prognostic signifi cance of number of positive nodes in cervical carcinoma stages IB, IIA, and IIB. Cancer 1990;65:1923-1927.

8. Sakuragi $N$ Satoh C, Takeda $N$, et al. Incidence and distribution pattern of pelvic and paraaortic lymph node metastasis in stages IB, IIA and IIB cervical carcinoma treated with radical hysterectomy. Cancer $1999 ; 85 ; 1547-1551$.

9. Morice $P$, Castaigne $D$, Patier $P$ et al. Interest at pelvic and paraaortic lymphadenectomy in patients with stage IB and II cervical carcinoma. Gynecol Oncol 1999:73:106-110.

10. Takeda $N$ Sakuragi $N$, Takeda $M$, et al. Multivariate analysis of histopathologic prognostic factors for invasive cervical cancer treated with radical hysterectomy and systematic retroperitoneal Iymphadenectomy. Acta Obstet Gynecol Scand 2002:81:1144-1151.

11. Boss EA, Barentsz JO, Massuger LFAG, ot al. The role of $M R$ imaging in invasive cervical carcinoma. Eur Radiol $2000 ; 10: 256-270$.

12. Bipat $S$, Glas $A S$, van der Velden $J$, et al. Computed tomography and magnetic resonance imaging in staging of uterine cervical carcinoma: a systematic review: Gynecol Oncol 2005:-91:59- 66 . 
I3. Chou HH, Chang $7 C$, Yen $7 \mathrm{C}$, et al. Low value of $118 \mathrm{FY}$. Alworg-3-deoxy-D-glucose positron emission tomography in primary staging of early-stage cervical cancer betore radical hysterectomy a Cin Oncol 2006.24:123 $12 B$

14. Levenback C. Burke TW. Gershenson DM, ot al, Intrapperative lymphatic mapping for vilvar cancer Obstet Gynecol 1994;81:163-167.

I5. Burke TW. Levenback C. Tornos C, et al. Intra abdontrial Iympharic mapping to direct selective pelvic and parasortic Ismphadenectomy in women with high. risk endonetrial cancer: results of a pilot study G3necol Oncol 1996;62:169: 173 .

16. Rob L, Strnad P. Robova H, et al. Study of lymphatic mapping and sentinel node identification in early stage cervical cancer. Gynecol Oncol 2005:98:281-288.

17. Marnitz $S$, Kohler $C$. Bongarft $S$, et al. German Association of Gynecologic Oncologists (ACO). Topographic distribution of sentinel lymph nodes in patients with cervical cancer Gynecol Oncol 2006;103;35-44.

18. Levenback C, Coleman RL, Burke TW, et at Lymphatic mapping and sentinel node identification in patients with cervix cancer undergoing radical hysterectomy and pelvic lymphadenectomy I Clin Oncol 2002;20:688-693.

19. Plante M, Renaud MC, Tetu B, et al. Laparoscopic sentinel node mapping in early stage cervical cancer Gynecol Oncol 2005:91:494-503.

20. Babu $M$, Haji AG, Kumar DKV et al. Sentinel node biopsy in cervicol cancer-A pllot study. Ind J Med \& Paed Oncology 2007:28:8-13.
S.V. S. Deo
Department of Surgical Oncology, IRCH All India Institute of Medical Sciences
New Delhi 